\title{
Nanoscale orientation mapping made easy: a new sample preparation workflow for rapid, large-area TKD analysis
}

Pat Trimby ${ }^{1}$, Iain Anderson ${ }^{2}$, John Lindsay ${ }^{1}$, Ali Gholinia ${ }^{3}$, Timothy Burnett ${ }^{4}$ and Philip Withers ${ }^{5}$

${ }^{1}$ Oxford Instruments, High Wycombe, England, United Kingdom, ${ }^{2}$ Oxford Instruments NanoAnalysis, United States, ${ }^{3}$ The University of Manchester, Manchester, England, United Kingdom, ${ }^{4}$ The University of Manchester, Manchester, United Kingdom, ${ }^{5}$ University of Manchester, Manchester, England, United Kingdom

The transmission Kikuchi diffraction (TKD) technique is a good example of how a scanning electron microscope (SEM) can be utilized for nanoscale analyses simply by working on electron transparent rather than bulk samples. Since the technique's development approximately 10 years ago [1, 2], TKD has been successfully applied to a wide range of materials and application fields, ranging from heavily deformed alloys to sub-micrometer inclusions in meteorites (e.g. [3]). The convenience of being able to utilize a conventional commercial electron backscatter diffraction (EBSD) system in a field emission SEM has made TKD a particularly attractive alternative to orientation mapping techniques in the transmission electron microscope (TEM). However, as with TEM, the requirement for an electron transparent sample is a significant drawback of the technique.

Many TKD studies of metals and alloys can utilize standard electropolishing preparation techniques, with 3 mm TEM discs providing large electron-transparent regions adjacent to the central perforation. However, focused ion beam (FIB) SEMs are more commonly used in applications where site-specific electrontransparent samples are required, a process that is both time consuming and, as it involves lift-out, susceptible to high failure rates. In addition, FIB-SEM lift-out samples are limited to relatively small sizes, typically less than 30 x $10 \square \mathrm{m}$, and often require low energy clean-up milling steps to remove unwanted ion-beam damage.

Here we introduce a new workflow for in-situ TKD sample preparation and analysis within a FIB-SEM. Unlike conventional approaches, this technique does not require any sample lift-out and the TKD analysis can follow on directly from the milling step, ideally without transfer to another SEM. The workflow requires an EBSDready top surface, which can be achieved using conventional bulk sample preparation techniques or can utilize a pristine, as-deposited surface layer such as is typical in thin films. Routine secondary electron (SE) or backscattered electron (BSE) imaging, as well as conventional EBSD analyses, can be carried out prior to (or even following) TKD sample preparation, as shown in Fig. 1. The following steps are then required for TKD sample preparation:

1. The sample is rotated so that the EBSD-ready surface faces downwards and can be aligned parallel to the milling source (e.g. an ion or laser beam) (Fig. 1, step 3).

2. Rapid milling of a sloping trench on the side of the sample, with the deepest part of the trench adjacent to the EBSD-ready surface, typically leaving a thickness of 1-5

3. Precision milling from the trench side to thin down the sample to electron transparency.

4. An optional further rotation of $10-20^{\circ}$ into an ideal orientation for off-axis TKD (Fig. 1, step 4).

This whole process can be carried out from start to finish in about 20-30 minutes using a Ga FIB-SEM. Since the TKD signal quality is determined by the nature of the exit (lower) surface, a high energy ion beam (e.g. 30 
$\mathrm{kV}$ ) can be used for the trench formation and sample thinning steps, with minimal concern for ion implantation or amorphization. Although in-situ techniques have been proposed for TEM sample preparation in the past [4], these are limited to pre-thinned samples (e.g. $30 \square \mathrm{m}$ thickness) and involve time-consuming milling through the whole sample thickness. Our approach is equally applicable on bulk samples, primarily because the subsequent off-axis TKD analysis can be successfully carried out with a backtilted sample geometry, necessitating milling only on the side of the sample.

The latest generation of plasma-FIB instruments and tri-beam systems equipped with a femtosecond laser are ideal for this new technique [5]. Not only can TKD samples then be prepared in a matter of a few minutes or less, but these fast-milling systems open the door to the routine preparation and analysis of large areas (e.g. $>100 \square \mathrm{m}$ field of view) with nanoscale resolution within the FIB-SEM. Here we will show example applications of this preparation workflow, from nanostructured surface films (such as the $\mathrm{HfO}_{2}$ film shown in Fig. 2) to large area samples prepared using a tri-beam system.

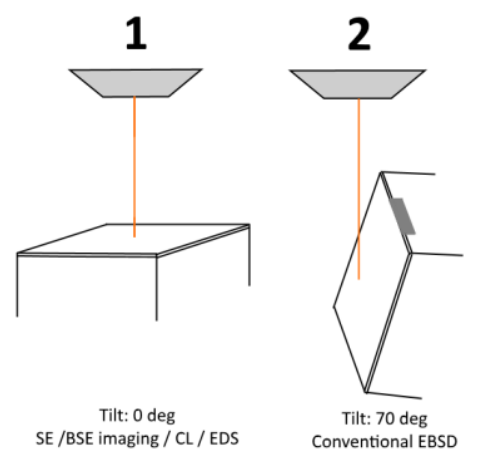

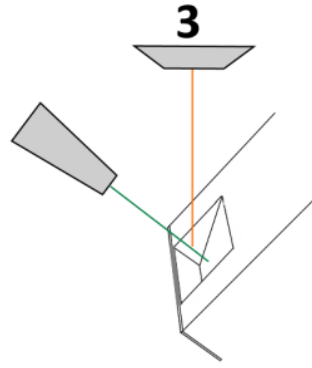

Tilt: $140-150 \mathrm{deg}$
FIB milling of sample side
4

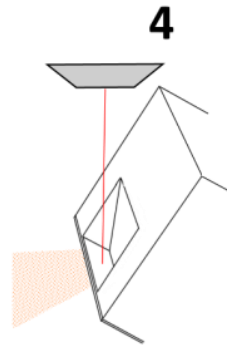

Tilt: $150-160$ deg TKD analysis of thinned region

Figure 1. Workflow steps for rapid TKD sample preparation. 1. Optional imaging and / or X-ray analysis of the surface of interest. 2. Optional conventional EBSD analysis. 3. Milling step with the surface of interest now facing downwards. A sloping trench is milled on the side of the bulk sample, with the thinned region milled down to the necessary electron transparency. 4. TKD analysis geometry - this can be carried out in the geometry used for step 3 or with the thinned sample area rotated to a lower tilt angle, as shown here.
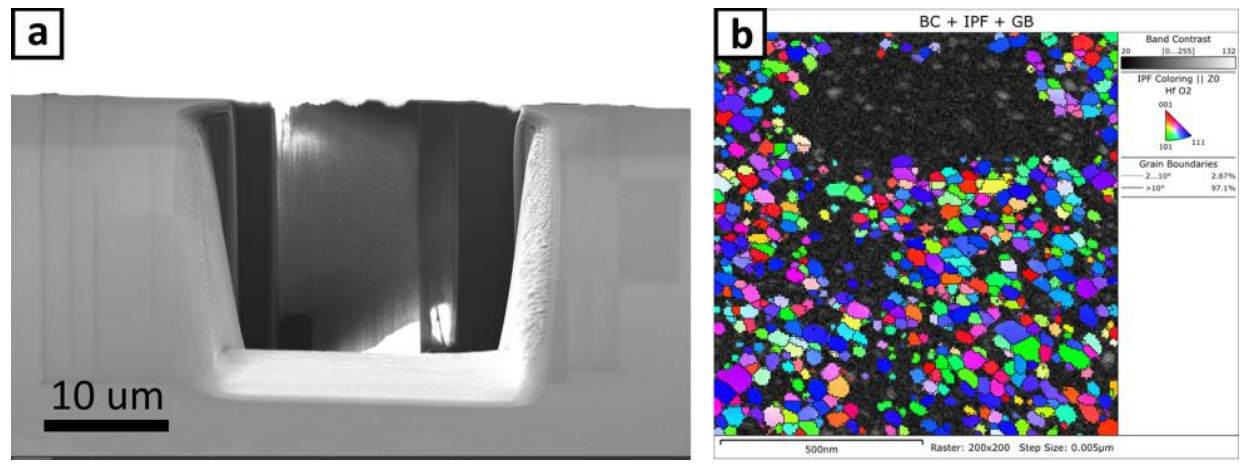

Figure 2. Example images from the rapid TKD workflow. (a) Typical electron beam image of the milled trench and the thinned sample region when oriented in the position for TKD analysis (step 4 in figure 1). (b) Example TKD orientation map of a nanostructured surface HfO2 film prepared for analysis in 20 minutes.

References

1. R.R. Keller and R.H. Geiss, J. Microscopy, 245 (2012), p. 245-251.

2. P.W. Trimby, Ultramicroscopy, 120 (2012), p. 16-24. 
3. G. Sneddon et al., Materials Science and Engineering R: Reports, 110 (2016), p. 1-12.

4. K. J. O'Shea et al., Micron, 66 (2014) p. 9-15.

5. M.P. Echlin et al., Current Opinion in Solid State and Materials Science 24 (2020) 100817. 